\title{
С.Л. Фуксон
}

\section{ОРТОГОНАЛЬНОСТИ ГРУППЫ $\mathbb{Z}_{p} \oplus \mathbb{Z}_{p}$}

\begin{abstract}
В марте 2013 г. International Journal of Mathematics and Mathematical Sciences опубликовал статью «Perpendicularity in an Abelian Group». Основная цель статьи - введение понятия бинарного отношения ортогональности в произвольной абелевой группе. Нами полностью исследованы прямые суммы групп $\mathbb{Z}_{3} \oplus \mathbb{Z}_{3}$ и $\mathbb{Z}_{5} \oplus \mathbb{Z}_{5}$, построены все их ортогональности. Также нам удалось обобщить полученные результаты для прямых сумм циклических групп $\mathbb{Z}_{p} \oplus \mathbb{Z}_{p}$.
\end{abstract}

Ключевые слова: ортогональность, группа, абелева группа, прямая сумма, циклическая группа.

Всё больше геометрические понятия проникают в пространство изучения алгебры. Haukkanen и другие [1] вводят понятие ортогональности в абелевой группе с помощью аксиом, которые оказываются вполне естественными, если придать им геометрическую интерпретацию.

Цель данного исследования - выяснить, какими ортогональностями обладает прямая сумма циклических групп $\mathbb{Z}_{p} \oplus \mathbb{Z}_{p}$. На пути к ответу на этот вопрос мы рассматриваем частные случаи: группы $\mathbb{Z}_{2} \oplus \mathbb{Z}_{2}, \mathbb{Z}_{3} \oplus \mathbb{Z}_{3}, \mathbb{Z}_{5} \oplus \mathbb{Z}_{5}$, и только после этого нам удаётся обобщить результаты для произвольной группы $\mathbb{Z}_{p} \oplus \mathbb{Z}_{p}$.

Пусть $G=(G,+)$ - аддитивная абелева группа. Пусть $\perp-$ бинарное отношение в $G$, удовлетворяющее следующим аксиомам:

(A1) $\forall a \in G: \exists b \in G: a \perp b$,

(A2) $\forall a \in G \backslash\{0\}: a \not \perp a$,

(A3) $\forall a, b \in G: a \perp b \Rightarrow b \perp a$,

(A4) $\forall a, b, \mathrm{c} \in G: a \perp b \wedge a \perp c \Rightarrow a \perp(b+c)$,

(A5) $\forall a, b \in G: a \perp b \Rightarrow a \perp-b$.

Будем называть данное бинарное отношение $\perp$ ортогональностью в $G$ [1].

Следующая ортогональность называется тривиальной:

$$
x \perp y \Leftrightarrow x=0 \vee y=0 .
$$

Однако наибольший интерес представляют нетривиальные ортогональности [1].

Определение 1. Назовём отношение ортогональности максимальной ортогональностью в $G$, если при добавлении к нему каких-либо новых пар полученное отношение уже не является ортогональностью в $G$.

Сформулированная ниже теорема помогает определить, когда $G$ имеет нетривиальную ортогональность. 
Теорема 2 [1]. Следующие условия эквивалентны:

(a) $G$ имеет нетривиальную ортогональность $\perp$;

(b) G имеет нетривиальные цииклческие подгруппь $H u K$, такие, что $H \cap K=\{0\}$

(c) G имеет нетривиальные подгруппь $H$ и $K$, такие, что $H \cap K=\{0\}$.

Наибольший интерес представляет собой доказательство импликации (c) $\Rightarrow$ (a), поскольку с его помощью вводится способ построения нетривиальных ортогональностей. А именно, определим $\perp$ как

$$
x \perp y \Leftrightarrow(x \in H \wedge y \in K) \vee(x \in K \wedge y \in H) \vee x=0 \vee y=0 .
$$

Пример 3. Пусть $G=\mathbb{Z}_{2} \oplus \mathbb{Z}_{2}$.

Обозначим через $0=(0,0), a=(0,1), b=(1,0), c=(1,1)$ элементы группы $\mathbb{Z}_{2} \oplus \mathbb{Z}_{2}$. Нетривиальными подгруппами группы $G$ являются подгруппы: $A=\{0$, $a\}, B=\{0, b\}, C=\{0, c\}$.

Применяя (2) к каждой паре подгрупп, получаем 3 нетривиальные ортогональности:

$$
\begin{aligned}
& x \perp y \Leftrightarrow(x \in A \wedge y \in B) \vee(x \in B \wedge y \in A) \vee x=0 \vee y=0, \\
& x \perp y \Leftrightarrow(x \in A \wedge y \in C) \vee(x \in C \wedge y \in A) \vee x=0 \vee y=0, \\
& x \perp y \Leftrightarrow(x \in B \wedge y \in C) \vee(x \in C \wedge y \in B) \vee x=0 \vee y=0 .
\end{aligned}
$$

Все эти ортогональности являются максимальными [1].

Оказывается, что ситуация существенно усложняется при рассмотрении группы $\mathbb{Z}_{3} \oplus \mathbb{Z}_{3}$.

Пример 4. Пусть $G=\mathbb{Z}_{3} \oplus \mathbb{Z}_{3}$.

$$
\begin{array}{rrr}
\mathbb{Z}_{3} \oplus \mathbb{Z}_{3}=\left\{0, a_{1}, a_{2}, a_{3}, a_{4}, a_{5}, a_{6}, a_{7}, a_{8}\right\}, \text { где } \\
0=(0,0), & a_{1}=(0,1), & a_{2}=(0,2), \\
a_{3}=(1,0), & a_{4}=(1,1), & a_{5}=(1,2), \\
a_{6}=(2,0), & a_{7}=(2,1), & a_{8}=(2,2) .
\end{array}
$$

В силу теоремы 2 , группа $G$ имеет нетривиальную ортогональность тогда и только тогда, когда $G$ имеет нетривиальные подгруппы, имеющие тривиальное пересечение. Так как порядок группы $\mathbb{Z}_{3} \oplus \mathbb{Z}_{3}$ равен 9, то, согласно теореме Лагранжа, порядок любой её нетривиальной подгруппы равен 3. Выпишем эти подгруппы:

$$
A=\left\{0, a_{1}, a_{2}\right\}, B=\left\{0, a_{3}, a_{6}\right\}, C=\left\{0, a_{4}, a_{8}\right\}, D=\left\{0, a_{5}, a_{7}\right\} .
$$

Заметим также, что каждые две подгруппы имеют лишь тривиальное пересечение. Таким образом, в силу (2), получаем 6 различных нетривиальных ортогональностей:

$$
\begin{aligned}
& x \perp_{1} y \Leftrightarrow(x \in A \wedge y \in B) \vee(x \in B \wedge y \in A) \vee x=0 \vee y=0, \\
& x \perp_{2} y \Leftrightarrow(x \in A \wedge y \in C) \vee(x \in C \wedge y \in A) \vee x=0 \vee y=0, \\
& x \perp_{3} y \Leftrightarrow(x \in A \wedge y \in D) \vee(x \in D \wedge y \in A) \vee x=0 \vee y=0, \\
& x \perp_{4} y \Leftrightarrow(x \in B \wedge y \in C) \vee(x \in C \wedge y \in B) \vee x=0 \vee y=0, \\
& x \perp_{5} y \Leftrightarrow(x \in B \wedge y \in D) \vee(x \in D \wedge y \in B) \vee x=0 \vee y=0, \\
& x \perp_{6} y \Leftrightarrow(x \in C \wedge y \in D) \vee(x \in D \wedge y \in C) \vee x=0 \vee y=0 .
\end{aligned}
$$


Будем называть такие ортогональности, полученные с помощью (2), элементарными.

Заметим, что каждая ортогональность соответствует паре подгрупп. Так, например, ортогональность $\perp_{1}$ соответствует паре $(A, B), \perp_{2}-$ паре $(A, C)$ и т.д.

Будем говорить, что $(X, Y)$ и $(S, T)$ не имеют общих элементов, если

$$
(X \cup Y) \cap(S \cup T)=0 .
$$

Например, $(A, B)$ и $(A, C)$ имеют общие элементы, а $(A, B)$ и $(C, D)$ нет.

Можно ли построить новые ортогональности, отличные от элементарных? Следующее предложение помогает при ответе на этот вопрос.

Предложение 5. Объединение ортогональностей, соответствующих парам подгрупп, не имеющих общих элементов, также является ортогональностью.

Таким образом, объединив ортогональности $\perp_{1}$ и $\perp_{6}, \perp_{2}$ и $\perp_{5}, \perp_{3}$ и $\perp_{4}$, мы получим 3 новые ортогональности:

$$
\begin{aligned}
& x \perp_{16} y \Leftrightarrow(x \in A \wedge y \in B) \vee(x \in B \wedge y \in A) \vee(x \in C \wedge y \in D) \vee \\
& \vee(x \in D \wedge y \in C) \vee x=0 \vee y=0, \\
& x \perp_{25} y \Leftrightarrow(x \in A \wedge y \in C) \vee(x \in C \wedge y \in A) \vee(x \in B \wedge y \in D) \vee \\
& \vee(x \in D \wedge y \in B) \vee x=0 \vee y=0, \\
& x \perp_{34} y \Leftrightarrow(x \in A \wedge y \in D) \vee(x \in D \wedge y \in A) \vee(x \in B \wedge y \in C) \vee \\
& \vee(x \in C \wedge y \in B) \vee x=0 \vee y=0 .
\end{aligned}
$$

Предложение 6. Ортогональности $\perp_{16}, \perp_{25}$ и $\perp_{34}$ являются максимальными.

Доказательство. Докажем, что $\perp_{16}$ является максимальной ортогональностью.

$$
\begin{gathered}
x \perp_{16} y \Leftrightarrow(x \in A \wedge y \in B) \vee(x \in B \wedge y \in A) \vee(x \in C \wedge y \in D) \vee \\
\vee(x \in D \wedge y \in C) \vee x=0 \vee y=0 .
\end{gathered}
$$

Предположим, что ортогональность $\perp_{16}$ не является максимальной, а значит, в силу определения максимальной ортогональности, $\perp_{16}$ можно расширить. По построению мы можем добавить пару, у которой или первая координата из $A$, а вторая из $C$, или первая координата из $B$, а вторая из $D$, или первая координата из $A$, а вторая из $D$, или первая координата из $B$, а вторая из $C$.

Рассмотрим случай, когда первая координата из $A$, а вторая из $C$. Остальные случаи рассматриваются аналогичным образом.

Пусть ортогональность $\perp$ такова, что

$$
\left(x \perp_{16} y\right) \vee\left(x=a_{1} \wedge y=a_{4}\right) \Rightarrow x \perp y,
$$

тогда, в силу (А4), из того, что $a_{1} \perp a_{4}$, следует $a_{1} \perp a_{8}$, где $a_{8}=a_{4}+a_{4} \in C$. Таким образом, $a_{1} \perp C$. Так как подгруппа $A$ циклическая и каждый её ненулевой элемент является её образующим, то

$$
A \perp C .
$$

Из (4) следует, что

$$
A \perp B \wedge C \perp D .
$$

Объединив условия (5) и (6), получим $A \perp B \wedge A \perp C \wedge C \perp D$. 
Воспользовавшись (А4), имеем $A \perp B \oplus C$, где $B \oplus C=G$. Таким образом, из того, что $A \subset G$, следует $A \perp A$, а значит, $a_{1} \perp a_{1} \wedge a_{2} \perp a_{2}$, что противоречит антирефлексивности ортогональности.

Таким образом, отношение $\perp$ не является ортогональностью.

Аналогичным образом можно показать, что ортогональности $\perp_{25}$ и $\perp_{34}$ также являются максимальными.

Замечание 7. Если пары подгрупп, соответствующие ортогональностям, имеют общие элементы, то их объединение не является ортогональностью.

Так, например, объединив $\perp_{1}$ и $\perp_{2}$, получим

$$
\begin{gathered}
x \perp_{12} y \Leftrightarrow(x \in A \wedge y \in B) \vee(x \in B \wedge y \in A) \vee(x \in A \wedge y \in C) \vee \\
\vee(x \in C \wedge y \in A) \vee x=0 \vee y=0 .
\end{gathered}
$$

Покажем, что отношение $\perp_{12}$ не является ортогональностью. Из определения $\perp_{12}$, в частности, вытекает, что $a_{1} \perp_{12} a_{3} \wedge a_{1} \perp_{12} a_{4}$. В силу (A4) $a_{1} \perp_{12} a_{3}+a_{4}$, то есть $a_{1} \perp_{12} a_{7}$, где $a_{7} \in D$, что противоречит (7).

Нетрудно показать, что всякая нетривиальная ортогональность в $\mathbb{Z}_{p} \oplus \mathbb{Z}_{p}$ может быть получена как объединение элементарных ортогональностей.

Таким образом, в рассматриваемой нами группе $\mathbb{Z}_{3} \oplus \mathbb{Z}_{3}$ существует 9 нетривиальных ортогональностей, 6 из которых являются элементарными, а 3 другие это всевозможные их объединения, являющиеся максимальными ортогональностями.

Перед тем как перейти к исследованию произвольной группы $\mathbb{Z}_{p} \oplus \mathbb{Z}_{p}$, рассмотрим группу $\mathbb{Z}_{5} \oplus \mathbb{Z}_{5}$.

Пример 8. Пусть $G=\mathbb{Z}_{5} \oplus \mathbb{Z}_{5}$.

$$
\begin{array}{rllll} 
& \mathbb{Z}_{5} \oplus \mathbb{Z}_{5}=\left\{0, a_{1}, a_{2}, \ldots, a_{24}\right\}, \text { где } & \\
0=(0,0), & a_{1}=(0,1), & a_{2}=(0,2), & a_{3}=(0,3), & a_{4}=(0,4), \\
a_{5}=(1,0), & a_{6}=(1,1), & a_{7}=(1,2), & a_{8}=(1,3), & a_{9}=(1,4), \\
a_{10}=(2,0), & a_{11}=(2,1), & a_{12}=(2,2), & a_{13}=(2,3), & a_{14}=(2,4), \\
a_{15}=(3,0), & a_{16}=(3,1), & a_{17}=(3,2), & a_{18}=(3,3), & a_{19}=(3,4), \\
a_{20}=(4,0), & a_{21}=(4,1), & a_{22}=(4,2), & a_{23}=(4,3), & a_{24}=(4,4) .
\end{array}
$$

Так как порядок группы $\mathbb{Z}_{5} \oplus \mathbb{Z}_{5}$ равен 25 , то порядок любой её нетривиальной подгруппы равен 5.

Выпишем эти подгруппы:

$$
\begin{gathered}
A=\left\{0, a_{1}, a_{2}, a_{3}, a_{4}\right\}, \\
B=\left\{0, a_{5}, a_{10}, a_{15}, a_{20}\right\}, \\
C=\left\{0, a_{6}, a_{12}, a_{18}, a_{24}\right\}, \\
D=\left\{0, a_{7}, a_{14}, a_{16}, a_{23}\right\}, \\
E=\left\{0, a_{8}, a_{11}, a_{19}, a_{22}\right\}, \\
F=\left\{0, a_{9}, a_{13}, a_{17}, a_{21}\right\} .
\end{gathered}
$$


С помощью (2) построим 15 элементарных ортогональностей, которые запишем в виде пар соответствующих им подгрупп:
1) $\perp_{1}:(A, B)$
$\perp_{2}:(A, C)$
$\perp_{3}:(A, D)$
$\perp_{4}:(A, E)$
2) $\perp_{6}:(B, C)$
$\perp_{7}:(B, D)$
$\perp_{8}:(B, E)$
$\perp_{9}:(B, F)$
3) $\perp_{10}:(C, D)$
$\perp_{11}:(C, E)$
$\perp_{12}:(C, F)$
4) $\perp_{13}:(D, E) \quad \perp_{14}:(D, F)$
5) $\perp_{15}:(E, F)$

Здесь в первой строке указаны пары, содержащие в качестве первой компоненты подгруппу $A$, во второй - подгруппу $B$ и так далее, пока не останется единственная пара $(E, F)$.

Рассмотрим ортогональности, являющиеся объединениями двух элементарных ортогональностей, удовлетворяющих (3).

Каждую из ортогональностей первой строки можно объединить с шестью другими ортогональностями; каждую ортогональность второй строки можно объединить с тремя из оставшихся ортогональностей; ортогональности третий строки объединяются с ортогональностями последующих строк единственным образом; ортогональности четвёртой и пятой строчек не могут быть объединены, поскольку имеют общие элементы. Запишем полученные ортогональности в следующую таблицу.

\begin{tabular}{|cccccc|ccc|c|}
\hline$\perp_{1,10}$ & $\perp_{1,11}$ & $\perp_{1,12}$ & $\perp_{1,13}$ & $\perp_{1,14}$ & $\perp_{1,15}$ & $\perp_{6,13}$ & $\perp_{6,14}$ & $\perp_{6,15}$ & \\
$\perp_{2,7}$ & $\perp_{2,8}$ & $\perp_{2,9}$ & $\perp_{2,13}$ & $\perp_{2,14}$ & $\perp_{2,15}$ & $\perp_{7,11}$ & $\perp_{7,12}$ & $\perp_{7,15}$ & $\perp_{10,15}$ \\
$\perp_{3,6}$ & $\perp_{3,8}$ & $\perp_{3,9}$ & $\perp_{3,11}$ & $\perp_{3,12}$ & $\perp_{3,15}$ & $\perp_{8,10}$ & $\perp_{8,12}$ & $\perp_{8,14}$ & $\perp_{11,14}$ \\
$\perp_{4,6}$ & $\perp_{4,7}$ & $\perp_{4,9}$ & $\perp_{4,10}$ & $\perp_{4,12}$ & $\perp_{4,14}$ & $\perp_{9,10}$ & $\perp_{9,11}$ & $\perp_{9,13}$ & $\perp_{12,13}$ \\
$\perp_{5,6}$ & $\perp_{5,7}$ & $\perp_{5,8}$ & $\perp_{5,10}$ & $\perp_{5,11}$ & $\perp_{5,13}$ & & & & \\
\hline
\end{tabular}

Таким образом, при объединении элементарных ортогональностей в пары возникает 45 более сложных ортогональностей. Однако все они не являются максимальными.

Объединим ортогональности в тройки. Для наглядности запишем их в виде соответствующих объединений пар подгрупп.

\begin{tabular}{|c|c|c|}
\hline$(A, B) \cup(C, D) \bigcup(E, F)$ & $(A, C) \cup(B, D) \cup(E, F)$ & $(A, D) \cup(B, C) \bigcup(E, F)$ \\
\hline$(A, B) \cup(C, E) \cup(D, F)$ & $(A, C) \cup(B, E) \cup(D, F)$ & $(A, D) \cup(B, E) \cup(C, F)$ \\
\hline$(A, B) \cup(C, F) \cup(D, E)$ & $(A, C) \cup(B, F) \cup(D, E)$ & $(A, D) \cup(B, F) \cup(C, E)$ \\
\hline \multicolumn{2}{|c|}{$(A, E) \cup(B, C) \cup(D, F)$} & $(A, F) \cup(B, C) \cup(D, E)$ \\
\hline \multicolumn{2}{|c|}{$(A, E) \bigcup(B, D) \bigcup(C, F)$} & $(A, F) \cup(B, D) \cup(C, E)$ \\
\hline \multicolumn{2}{|c|}{$(A, E) \cup(B, F) \cup(C, D)$} & $(A, F) \cup(B, E) \cup(C, D)$ \\
\hline
\end{tabular}

Аналогично предложению 6 показывается, что все эти 15 ортогональностей максимальны. При объединении элементарных ортогональностей по четыре возникающие отношения уже не являются ортогональностями, а значит, группа $\mathbb{Z}_{5} \oplus \mathbb{Z}_{5}$ имеет ровно 75 нетривиальных ортогональностей. 
Обобщим полученные результаты. Рассмотрим произвольную группу $\mathbb{Z}_{p} \oplus \mathbb{Z}_{p}$, где $p \geq 3, p-$ простое число:

$$
\begin{gathered}
\mathbb{Z}_{p}=\{0,1,2, \ldots, p-1\}, \\
\mathbb{Z}_{p} \oplus \mathbb{Z}_{p}=\left\{0, a_{1}, a_{2}, \ldots, a_{p^{2}-1}\right\} .
\end{gathered}
$$

Порядок любой нетривиальной подгруппы группы $\mathbb{Z}_{p} \oplus \mathbb{Z}_{p}$ равен $p$. Так как каждая нетривиальная подгруппа группы $\mathbb{Z}_{p} \oplus \mathbb{Z}_{p}$ циклическая и содержит $p-1$ ненулевых элементов (каждый из которых является её образующим), то количество её подгрупп равно числу

$$
\frac{p^{2}-1}{p-1}=p+1 \text {. }
$$

Таким образом, можно построить $C_{p+1}^{2}$ элементарных ортогональностей:

$p$ ортогональностей, соответствующих парам $(A, X)$, где $X$ - произвольная нетривиальная подгруппа, $X \neq A$;

$(p-1)$ ортогональностей, соответствующих парам $(B, X)$, где $X$ - произвольная нетривиальная подгруппа, $X \neq A, B$;

одну ортогональность, соответствующую паре двух последних подгрупп.

Теорема 9. Число $s$ всевозможных нетривиальных ортогональностей прямой суммы циклических групп $\mathbb{Z}_{p} \oplus \mathbb{Z}_{p}, p \geq 3$, вычисляется по формуле

$$
S=\sum_{i=0}^{\frac{p-1}{2}} \frac{C_{p+1}^{2} \cdot C_{p-1}^{2} \cdot \ldots \cdot C_{p-(2 i-1)}^{2}}{(i+1) !}
$$

Доказательство. Число элементарных ортогональностей $\mathbb{Z}_{p} \oplus \mathbb{Z}_{p}$ равно $C_{p+1}^{2}$. Объединим элементарные ортогональности в пары так, чтобы полученные отношения снова были ортогональностями. Выберем из $C_{p+1}^{2}$ пару ортогональностей, удовлетворяющую (3). Первую ортогональность можно выбрать $C_{p+1}^{2}$ способами, вторую - $C_{p-1}^{2}$ способами, исключив ортогональности, которые соответствуют парам подгрупп, содержащих в качестве хотя бы одной из компонент подгруппы из первой ортогональности. Так как $(X, Y) \cup(S, T)=(S, T) \cup(X, Y)$, то формула для подсчёта ортогональностей, являющихся объединениями двух элементарных, имеет следующий вид:

$$
\frac{C_{p+1}^{2} \cdot C_{p-1}^{2}}{2 !} .
$$

С помощью аналогичных рассуждений можно получить формулу для подсчёта числа ортогональностей, соответствующих объединению максимального возможного числа элементарных ортогональностей в тройки:

$$
\frac{C_{p+1}^{2} \cdot C_{p-1}^{2} \cdot C_{p-3}^{2}}{3 !}
$$


Продолжая этот процесс, получим в конце формулу для вычисления числа ортогональностей, соответствующих объединению максимально возможного числа элементарных ортогональностей с соблюдением условия (3). При добавлении к таким ортогональностям новой элементарной ортогональности условие (3) уже не будет выполняться и полученное отношение не будет ортогональностью. Формула имеет вид

$$
\frac{C_{p+1}^{2} \cdot C_{p-1}^{2} \cdot C_{p-3}^{2} \cdot \ldots \cdot C_{4}^{2} \cdot C_{2}^{2}}{\left(\frac{p+1}{2}\right) !} .
$$

Таким образом, последняя формула соответствует числу максимальных ортогональностей группы $\mathbb{Z}_{p} \oplus \mathbb{Z}_{p}$, где $p \geq 3$.

Просуммировав полученные результаты, имеем формулу для вычисления числа всевозможных нетривиальных ортогональностей прямой суммы $\mathbb{Z}_{p} \oplus \mathbb{Z}_{p}, p \geq 3$ :

$$
\sum_{i=0}^{\frac{p-1}{2}} \frac{C_{p+1}^{2} \cdot C_{p-1}^{2} \cdot \ldots \cdot C_{p-(2 i-1)}^{2}}{(i+1) !}
$$

Для наглядности занесём результаты, полученные для всех исследуемых нами групп, в таблицу.

\begin{tabular}{|c|c|c|c|}
\hline Группа & $\begin{array}{c}\text { Число } \\
\text { элементарных } \\
\text { ортогональностей }\end{array}$ & $\begin{array}{c}\text { Число максимальных } \\
\text { ортогональностей }\end{array}$ & $\begin{array}{c}\text { Число всевозможных } \\
\text { нетривиальных } \\
\text { ортогональностей }\end{array}$ \\
\hline $\mathbb{Z}_{2} \oplus \mathbb{Z}_{2}$ & 3 & 3 & 3 \\
\hline $\mathbb{Z}_{3} \oplus \mathbb{Z}_{3}$ & 6 & 3 & 9 \\
\hline $\mathbb{Z}_{5} \oplus \mathbb{Z}_{5}$ & 15 & 15 & 75 \\
\hline $\mathbb{Z}_{p} \oplus \mathbb{Z}_{p}$, \\
$p \geq 3$
\end{tabular}

Таким образом, нам удалось полностью описать ортогональности группы $\mathbb{Z}_{p} \oplus \mathbb{Z}_{p}$ и найти их число.

\section{ЛИТЕРАТУРА}

1. Haukkanen P., Mattila M., Merikoski J.K., Tossavainen T. Perpendicularity in an Abelian group // International Journal of Mathematics and Mathematical Sciences. 2013. V. 13.

2. Haukkanen P., Merikoski J.K., Tossavainen T. Axiomatizing perpendicularity and parallelism // Journal for Geometry and Graphics. 2011. V. 15. No. 2. P. 129-139.

3. Davis G. Rings with orthogonality relation // Bulletin of the Australian Mathematical Society. 1971. V. 4. P. 163-178.

4. Векслер А.И. Linear spaces with disjoint elements and their conversion into vector lattices // Учёные записки Ленинградского государственного педагогического института имени А.И. Герцена. 1967. Вып. 328. С. 19-43. 


\section{Fukson S. L. ORTHOGONALITIES IN $\mathbb{Z}_{p} \oplus \mathbb{Z}_{p}$}

\section{DOI $10.17223 / 19988621 / 36 / 6$}

More and more geometric concepts penetrate into the space of the study of algebra. On March 2013, the International Journal of Mathematics and Mathematical Sciences carried an article titled "Perpendicularity in an Abelian Group" written by Haukkanen et al. The main objective of that article was to introduce the concept of a binary relation of orthogonality in an arbitrary Abelian group. The ratio of orthogonality in different algebraic structures has already aroused the interest of mathematicians before. For example, orthogonality in the rings was studied by Davis in the paper "Rings with orthogonality relation" (Bulletin of the Australian Mathematical Society, vol. 4, 1971), Veksler considers orthogonality in lattices and lattice-ordered groups in "Linear spaces with disjoint elements and their conversion into vector lattices" (Leningrad. Gos. Ped. Inst. Uchen. Zap., vol. 328, pp. 19-43, 1967). The purpose of the present paper is to get some results about orthogonalities for specific Abelian groups. We have investigated in detail the orthogonalities of a direct sum of cyclic groups $\mathbb{Z}_{p} \oplus \mathbb{Z}_{p}$. On the way to answer how many and which orthogonalities this group has, we consider special cases, namely, $\mathbb{Z}_{3} \oplus \mathbb{Z}_{3}, \mathbb{Z}_{5} \oplus \mathbb{Z}_{5}$ and only after that we are able to summarize the results to an arbitrary group $\mathbb{Z}_{p} \oplus \mathbb{Z}_{p}$.

Haukkanen and others introduced the concept of orthogonality in an Abelian group with the help of axioms which are absolutely natural if we give a geometric interpretation to them.

Let $G=(G,+)$ be an additive Abelian Group. Let $\perp$ be a binary relation in $G$ satisfying:

(A1) $\forall a \in G: \exists b \in G: a \perp b$,

(A2) $\forall a \in G \backslash\{0\}: a \not \subset a$,

(A3) $\forall a, b \in G: a \perp b \Rightarrow b \perp a$,

(A4) $\forall a, b, \mathrm{c} \in G: a \perp b \wedge a \perp c \Rightarrow a \perp(b+c)$,

(A5) $\forall a, b \in G: a \perp b \Rightarrow a \perp-b$.

We call $\perp$ an orthogonality in $G$.

Definition. We call $\perp$ maximal orthogonality in $G$ if it satisfies the axioms (A1) - (A5) and after adding thereto any of the other pair, the ratio obtained is not orthogonality in $G$.

We explore orthogonalities of groups $\mathbb{Z}_{3} \oplus \mathbb{Z}_{3}, \mathbb{Z}_{5} \oplus \mathbb{Z}_{5}$ and consider the general case of a direct sum $\mathbb{Z}_{p} \oplus \mathbb{Z}_{p}$. With the help of our research, we were able to find the number of elementary, maximal, and all possible orthogonalities in the studied groups.

We present our results in the following table.

\begin{tabular}{|c|c|c|c|}
\hline Group & $\begin{array}{c}\text { The number } \\
\text { of elementary } \\
\text { orthogonalities }\end{array}$ & $\begin{array}{c}\text { The number } \\
\text { of maximal orthogonalities }\end{array}$ & $\begin{array}{c}\text { The number of all possible } \\
\text { orthogonalities }\end{array}$ \\
\hline $\mathbb{Z}_{2} \oplus \mathbb{Z}_{2}$ & 3 & 3 & 3 \\
\hline $\mathbb{Z}_{3} \oplus \mathbb{Z}_{3}$ & 6 & 3 & 9 \\
\hline $\mathbb{Z}_{5} \oplus \mathbb{Z}_{5}$ & 15 & 15 & 75 \\
\hline $\mathbb{Z}_{p} \oplus \mathbb{Z}_{p}$, \\
$p \geq 3$
\end{tabular}

Keywords: Orthogonality, group, Abelian group, direct sum, cyclic group

\section{FUKSON Sofia Leonidovna (Tomsk State University, Tomsk, Russian Federation)}

E-mail: fouk.son.ya@gmail.com 


\section{REFERENCES}

1. Haukkanen P., Mattila M., Merikoski J.K., Tossavainen T. Perpendicularity in an Abelian group. International Journal of Mathematics and Mathematical Sciences, 2013, vol. 13.

2. Haukkanen P., Merikoski J.K., Tossavainen T. Axiomatizing perpendicularity and parallelism. Journal for Geometry and Graphics, 2011, vol. 15, no. 2, pp. 129-139.

3. Davis G. Rings with orthogonality relation. Bulletin of the Australian Mathematical Society, 1971, vol. 4, pp. 163-178.

4. Veksler A.I. Linear spaces with disjoint elements and their conversion into vector lattices. Uchenye zapiski Leningradskogo gosudarstvennogo pedagogicheskogo instituta imeni A.I. Gertsena, 1967, no. 328, pp. 19-43. (in Russian) 